\title{
Non-Fermi-liquid phases in the two-band Hubbard model: Finite-temperature exact diagonalization study of Hund's rule coupling
}

\author{
A. Liebsch and T. A. Costi \\ Institut für Festkörperforschung, Forschungszentrum Jülich, 52425 Jülich, Germany
}

\begin{abstract}
The two-band Hubbard model involving subbands of different widths is investigated via finitetemperature exact diagonalization (ED) and dynamical mean field theory (DMFT). In contrast to the quantum Monte Carlo (QMC) method which at low temperatures includes only Ising-like exchange interactions to avoid sign problems, ED permits a treatment of Hund's exchange and other onsite Coulomb interactions on the same footing. The role of finite-size effects caused by the limited number of bath levels in this scheme is studied by analyzing the low-frequency behavior of the subband self-energies as a function of temperature, and by comparing with numerical renormalization group (NRG) results for an effective one-band model. For half-filled, non-hybridizing bands, the metallic and insulating phases are separated by an intermediate mixed phase with an insulating narrow and a bad-metallic wide subband. The wide band in this phase exhibits different degrees of non-Fermi-liquid behavior, depending on the treatment of exchange interactions. Whereas for complete Hund's coupling, infinite lifetime is found at the Fermi level, in the absence of spin-flip and pair-exchange, this lifetime becomes finite. Excellent agreement is obtained both with new NRG and previous QMC/DMFT calculations. These results suggest that-finite temperature ED/DMFT might be a useful scheme for realistic multi-band materials.
\end{abstract}

PACS. 71.20.Be Transition metals and alloys - 71.27+a Strongly correlated electron systems

\section{INTRODUCTION}

Strongly correlated materials exhibit a wealth of fascinating physical phenomena associated with complex single-electron and many-electron interactions. Transition metal oxides, for example, tend to have partially filled shells of highly correlated $d$ electrons, surrounded by complicated lattice geometries, with many atoms and electrons per unit cell. The theoretical description of the electronic properties of these materials is a challenging topic in condensed matter physics. Significant advances were achieved during recent years via the dynamical mean field theory (DMFT) 1, 2] which provides a treatment of single-electron and many-electron interactions on the same footing.

For realistic materials, DMFT has been used extensively in combination with the quantum Monte Carlo (QMC) method 3]. This approach has the advantage that it can be readily applied to systems consisting of two or more subbands. It has the drawback, however, that, to avoid sign problems at low temperatures, it includes only Ising-like exchange interactions [4]. Improvements of the QMC method to include the full Hund's coupling are so far limited to $T=0$ or rather high temperatures [5, 6, 7]. In view of this limitation there is clearly a need to explore alternative methods that are applicable to multi-band materials and complete onsite exchange interactions.

The aim of the present work is two-fold: First, exact diagonalization (ED) [1, 8] is proposed as a potentially highly useful impurity solver for finite- $T$ DMFT studies of realistic systems. The attractive feature of this approach is that, in contrast to finite- $T$ QMC, on- site Coulomb and exchange interactions are treated on the same basis. Second, we apply finite- $T$ ED/DMFT to a highly nontrivial system which has recently received considerable attention, namely, the two-band Hubbard model consisting of subbands of different widths 5, 6, 9, 10, 11, 12, 13, 14, 15, 16, 17, 18, 19, 20, 21]. The phase diagram of this system was recently evaluated in Refs. 16, 17. Here we focus on the electronic properties of the so-called orbital-selective phase in which the narrow band is insulating while the wide band is still metallic. To examine the influence of finite-size effects in the ED approach, and to investigate the electronic properties in the limit of low temperatures, we have also performed numerical renormalization group (NRG) DMFT calculations for a simplified two-band model which is particularly suited for the intermediate phase.

The main result of this paper is that the two-band ED/DMFT calculations provide a correct picture of the electronic properties of the Hubbard model involving nonequivalent subbands, including the unusual nonFermi-liquid properties of the orbital-selective phase. This outcome is remarkable since, for computational reasons, the number of bath levels per impurity orbital is necessarily smaller than in analogous one-band models. Nevertheless, despite this limitation, the ED results are in good qualitative or, in some cases, quantitative agreement with the NRG results. In the Ising case they also agree very well with previous QMC/DMFT results [13, 21].

The overall consistency with the NRG and QMC calculations suggests that finite- $T$ ED/DMFT might indeed be very useful in the future to analyze realistic materials, especially, if full diagonalization is replaced by finite- $T$ 
Lanczos methods 22, 23] in order to be able to deal with larger cluster sizes.

Hubbard models involving nonequivalent subbands are relevant for compounds such as $\mathrm{Ca}_{2-x} \mathrm{Sr}_{x} \mathrm{RuO}_{4}$ and $\mathrm{Na}_{x} \mathrm{CoO}_{2}$, where, as a result of the nearly twodimensional lattice structure, coexisting narrow and wide bands arise naturally. Thus, onsite Coulomb energies can be simultaneously large and small relative to the widths of important subbands. As a function of doping concentration, both materials give rise to a remarkably rich sequence of phases, including superconductivity and Mott insulating behavior 24, 25]. Evidently, the competition between multiple kinetic energy scales and local Coulomb and exchange energies is an important feature of these strongly correlated systems.

A consistent treatment of Coulomb and exchange interactions in materials of this kind is important since it has recently become clear that the Hund's coupling has a decisive influence on the nature of the Mott transition 5, 14, 16, 18, 19, 26]. In fact, the different treatments of exchange terms in earlier finite- $T$ QMC 10, 11, 13. and zero- $T$ ED [12] calculations have given rise to some confusion, with apparently contradictory results. As was clarified in Ref. [14] for $T=0$ and in Ref. [16] for $T>0$, however, the QMC and ED results are in agreement provided that exchange interactions are treated in the same manner. Thus, for full Hund's coupling the two-band Hubbard model exhibits successive first-order transitions. In striking contrast, in the absence of spin-flip and pairexchange only the lower transition remains first-order [13, 16, 20].

Moreover, as will be discussed in detail below, the nature of the intermediate phase depends in a subtle manner on the treatment of exchange interactions. In this regard the ED and NRG results yield the following picture: For full Hund's coupling, the wide band has infinite lifetime at $E_{F}$ but does not satisfy Fermi-liquid criteria at finite frequencies. This finding is consistent with recent results obtained by Biermann et al. 21] in $T=0$ two-band ED/DMFT calculations. For Ising exchange, instead, correlations are significantly enhanced and the lifetime becomes finite even at $E_{F}$, in agreement with QMC calculations 13, 21].

Thus, the Mott transition in the Hubbard model involving different subbands does not consist of equivalent sequential transitions. Instead, when the narrow band becomes insulating, the wide band is forced into a badmetallic state whose deviations from Fermi-liquid behavior depend in a qualitative manner on the treatment of exchange interactions.

In the past, finite- $T$ ED/DMFT methods have been applied mainly to single-band cases, where the incorporation of an appropriate cluster of bath levels is feasible. Throughout this paper we consider two impurity levels, each surrounded by either 2 or 3 bath levels, giving total cluster sizes $n_{s}=6$ or $n_{s}=8$ per spin. Below we demon- strate that even a cluster size of $n_{s}=6$ provides qualitatively correct subband self-energies. For instance, at $T=10 \mathrm{meV}$ the critical Coulomb energies of the orbitalselective Mott transitions for $n_{s}=6$ differ by only about $0.1 \ldots 0.2 \mathrm{eV}$ from those derived for $n_{s}=8$. This finding is interesting since it suggests that finite- $T$ ED/DMFT calculations for more realistic three-band models using a cluster size of $n_{s}=9$ (three impurity levels, each coupled to two bath levels) should be useful. This would allow one to re-examine the Mott transition in systems that have been studied previously using QMC/DMFT for Ising exchange interactions.

A more accurate representation of low-frequency properties at low temperatures requires three bath levels per impurity orbital: one near the Fermi level to provide adequate metallicity, and two for the upper and lower Hubbard bands, giving $n_{s}=8$ per spin. This extension leads to a significant reduction of finite-size effects. Several comparisons of results for $n_{s}=6$ and $n_{s}=8$ are provided below to illustrate the range of applicability of the two-band ED/DMFT approach.

The outline of the paper is as follows: In Section 2 the multiband Hubbard model is specified and its numerical solution via the finite- $T$ exact diagonalization method is discussed. Section 3 describes the effective one-band model used in the NRG approach to evaluate the electronic properties of the wide band in the intermediate phase when the narrow band is insulating. In Section 4 the ED/DMFT is applied to the purely metallic phase just below the first Mott transition. Section 5 deals with the intermediate phase in the presence of full Hund's coupling, where wide band exhibits infinite lifetime at $E_{F}$, combined with non-Fermi-liquid behavior at finite frequencies. In Section 6 this intermediate phase is considered in the absence of spin-flip and pair-exchange terms, giving rise to finite lifetime even at $E_{F}$. In Section 7 the two-band ED approach is applied to the case studied previously within QMC/DMFT 13. Section 8 presents analogous results for the case considered recently within QMC/DMFT by Biermann et al. 21. Section 9 contains a brief discussion of iterated perturbation theory (IPT) DMFT results for the two-band model and Section 10 provides a summary and outlook.

\section{MULTIBAND EXACT DIAGONALIZATION METHOD}

The two-band Hubbard model for non-equivalent subbands is represented by the Hamiltonian:

$$
\begin{aligned}
H & =H_{0}+H_{1}+H_{2} \\
H_{0} & =\sum_{l m i \sigma} t_{l m i} c_{l i \sigma}^{+} c_{m i \sigma} \\
H_{1} & =\sum_{l i} U n_{l i \uparrow} n_{l i \downarrow}+\sum_{l \sigma \sigma^{\prime}}\left(U^{\prime}-J \delta_{\sigma \sigma^{\prime}}\right) n_{l 1 \sigma} n_{l 2 \sigma^{\prime}}
\end{aligned}
$$




$$
\begin{aligned}
H_{2}= & -J^{\prime} \sum_{l}\left[c_{l 1 \uparrow}^{+} c_{l 1 \downarrow} c_{l 2 \downarrow}^{+} c_{l 2 \uparrow}+\text { H.c. }\right] \\
& -J^{\prime} \sum_{l}\left[c_{l 1 \uparrow}^{+} c_{l 1 \downarrow}^{+} c_{l 2 \uparrow} c_{l 2 \downarrow}+\text { H.c. }\right]
\end{aligned}
$$

where $c_{l i \sigma}^{+}$and $c_{l i \sigma}$ are creation and annihilation operators for electrons at site $l$ in orbital $i=1,2$ with spin $\sigma$ and $n_{l i \sigma}=c_{l i \sigma}^{+} c_{l i \sigma}$. H.c. denotes Hermitian conjugate terms. $H_{0}$ is the single-particle Hamiltonian which we represent by half-filled, non-hybridizing bands with density of states $N_{i}(\omega)=2 /\left(\pi D_{i}\right) \sqrt{1-\left(\omega / D_{i}\right)^{2}}$ and widths $W_{1}=2 \mathrm{eV}, W_{2}=4 \mathrm{eV}$, where $W_{i}=2 D_{i}$. $H_{1}$ represents the anisotropic, Ising-like onsite Coulomb and exchange interactions and $\mathrm{H}_{2}$ additional spin-flip and pairexchange contributions. For isotropic Hund's coupling one has $J^{\prime}=J=\left(U-U^{\prime}\right) / 2$. Below, we discuss results for $J^{\prime}=J$ as well as $J^{\prime}=0$. The latter case corresponds to the Ising-like exchange treatment in previous QMC/DMFT calculations [11, 13].

The ED/DMFT results are derived from a two-band generalization of the approach employed for single bands [1, 8]. The effective Anderson impurity Hamiltonian includes impurity levels $\epsilon_{1,2}$ and bath levels $\epsilon_{k=3 \ldots n_{s}}$ (see Fig. 1). For particle-hole symmetry $\epsilon_{1,2}=0$. Each impurity level interacts with its own bath, so that for $n_{s}=6$ impurity level 1 couples to bath levels 3,4 with $\epsilon_{4}=-\epsilon_{3}$, while level 2 couples to bath levels 5,6 with $\epsilon_{6}=-\epsilon_{5}$. For $n_{s}=8$ impurity levels 1,2 couple in addition to the bath levels $\epsilon_{7}=0$ and $\epsilon_{8}=0$, respectively.

Since at $T>0$ all states of the impurity Hamiltonian are used in the construction of the subband Green's functions, for $n_{s}=6$ the largest matrix to be diagonalized corresponds to the sector $n_{\uparrow}=n_{\downarrow}=3$, with dimension $\left[n_{s} ! /\left(\left(n_{s} / 2\right) !\right)^{2}\right]^{2}=400$. For $n_{s}=8$ this dimension increases to 4900 . We neglect hybridization between bands, so that self-energies and Green's functions are diagonal in orbital space. Denoting eigenvalues and eigenvectors of the impurity Hamiltonian by $E_{\nu}$ and $|\nu\rangle$, the subband

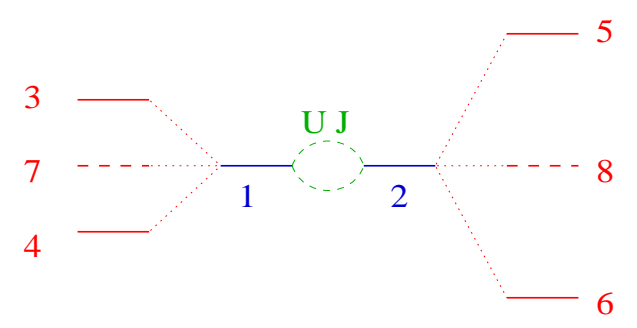

FIG. 1: Level diagram for ED scheme. Blue lines: impurity levels; red solid (plus dashed) lines: bath levels for $n_{s}=6$ $\left(n_{s}=8\right)$. Green lines: Coulomb and exchange interactions between impurity levels; red dotted lines: hopping interactions between impurity and bath levels.
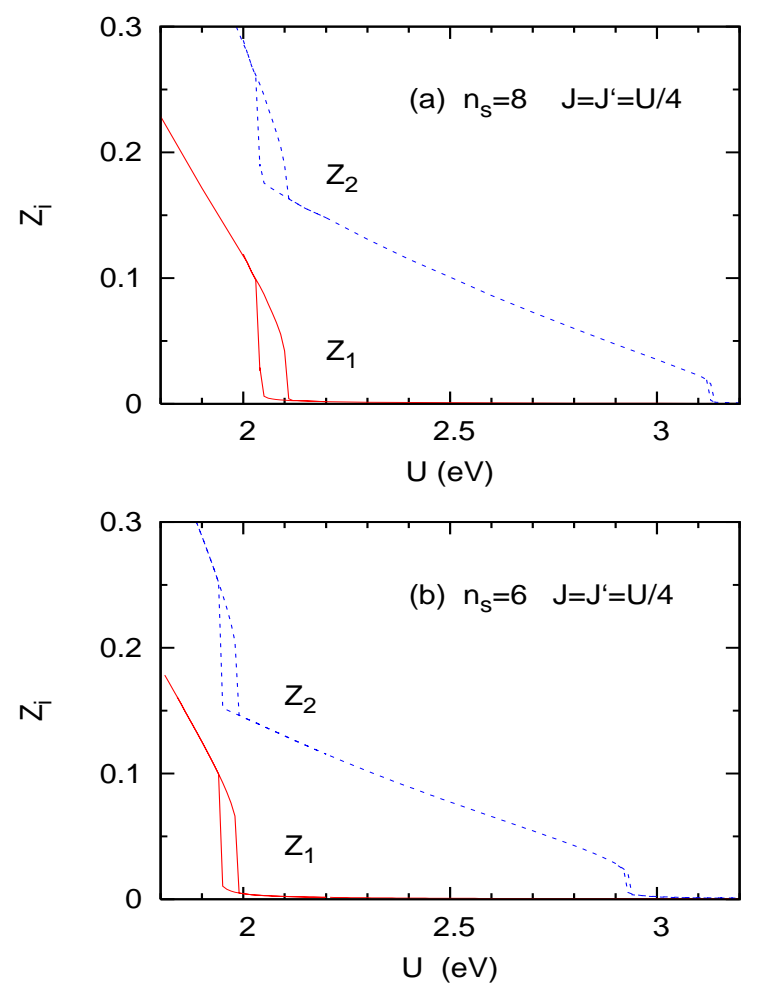

FIG. 2: $\quad Z_{i}(U)$ as a function of $U$ for $J=J^{\prime}=U / 4$ at $T=$ $10 \mathrm{meV}$, calculated within ED/DMFT for different cluster sizes: (a) $n_{s}=8$; (b) $n_{s}=6$. Solid (red) curves: narrow band, dashed (blue) curves: wide band.

Green's functions are evaluated from the expression

$$
G_{i}\left(i \omega_{n}\right)=\frac{1}{Z} \sum_{\nu \mu} \frac{\left|\left\langle\nu\left|c_{0 i \sigma}^{+}\right| \mu\right\rangle\right|^{2}}{E_{\nu}-E_{\mu}-i \omega_{n}}\left[e^{-\beta E_{\nu}}+e^{-\beta E_{\mu}}\right] .
$$

Here, $\beta=1 / k_{B} T, \omega_{n}=(2 n+1) \pi / \beta$ are Matsubara frequencies, and $Z=\sum_{\nu} \exp \left(-\beta E_{\nu}\right)$ is the partition function. $l=0$ denotes the impurity site. Since we consider only paramagnetic phases the spin index of Green's functions and self-energies is omitted for convenience. Because of the particle-hole symmetry of the present system, Green's functions and self-energies are purely imaginary quantities.

To provide an overview of the Mott transitions in this model and to illustrate the sensitivity of the critical Coulomb energies to the cluster size used in the ED/DMFT, we show in Fig. 2 the quantities $Z_{i}(U)=$ $1 /\left[1-\operatorname{Im} \Sigma_{i}\left(i \omega_{0}\right) / \omega_{0}\right]$ for $n_{s}=6$ and $n_{s}=8$ at $T=$ $10 \mathrm{meV}$, assuming $J^{\prime}=J=U / 4$. Here $\Sigma_{i}\left(i \omega_{0}\right)$ are the subband self-energies at the first Matsubara frequency. Thus, for isotropic Hund's exchange and $n_{s}=8$, $U_{c 1} \approx 2.1 \mathrm{eV}$ and $U_{c 2} \approx 3.1 \mathrm{eV}$. (In our notation the $U_{c i=1,2}$ refer here to the subband critical Coulomb energies for increasing $U$ and should not be confused with the stability boundaries for increasing vs decreasing $U$ at the individual Mott transitions.) Near both critical Coulomb 
energies $Z_{i}(U)$ exhibit hysteresis behavior characteristic of first-order transitions. For $n_{s}=6$, the common metallic phase is stable only up to about $U_{c 1} \approx 2 \mathrm{eV}$. Thus, inclusion of zero-energy bath levels supports the metallic character of the DMFT solution. A shift of about $0.2 \mathrm{eV}$ is found for $U_{c 2}$ where the wide band becomes insulating. These shifts are consistent with single-band ED/DMFT results 16] upon increasing $n_{s}$ from 3 to 4; only minor additional shifts occur in this case between $n_{s}=4$ and the fully converged results for $n_{s}=6$.

According to the QMC/DMFT results discussed in Ref. [13], the Mott transitions for $J=U / 4$ and $J^{\prime}=0$, i.e., for Ising-like exchange, are located at $U_{c 1} \approx 2.1 \mathrm{eV}$ and $U_{c 2} \approx 2.7 \mathrm{eV}$. Moreover, apart from the shift of $U_{c 2}$, this transition is no longer first-order. The two-band ED/DMFT results in Ref. 16 for $n_{s}=6$ confirmed this fundamental difference between the $J^{\prime}=J$ and $J^{\prime}=0$ treatments.

As will be discussed in detail below, the wide band in the intermediate phase, i.e., for $U_{c 1}<U<U_{c 2}$, does not satisfy Fermi-liquid criteria. Thus, the fact that $Z_{2}(U)$ as defined above is finite in this region does not imply existence of ordinary quasiparticles. In the case of full Hund's coupling, the imaginary part of the self-energy of the wide band vanishes in the low-frequency limit, but does not increase linearly at small $i \omega_{n}$, implying nonquadratic variation at real frequencies. For Ising-like exchange, $\operatorname{Im} \Sigma_{2}\left(i \omega_{n}\right)$ remains finite for $\omega_{n} \rightarrow 0$, implying finite lifetime even for states at $E_{F}$. Plots like those in Fig. 1 are nevertheless useful since they permit a convenient identification of phase transitions. Thus, $Z_{i}(U) \rightarrow 0$ at $U_{c i}$ indicates that the narrow or wide subbands become insulating for $U>U_{c i}$, respectively. (Of course, due to the discrete representation along the imaginary frequency axis at finite $T, Z_{i}(U)$ does not fully vanish.)

To check the convergence of the ED results with cluster size $n_{s}$ we compare them with NRG calculations for an effective one-band model which is most suitable for the intermediate phase and which is specified in the following section. Since the narrow band is insulating in this phase one of the key features of the effective model is the omission of one-electron hopping in the narrow band. Accordingly, the upper and lower Hubbard peaks will be centered at about $\pm U / 2$, but the influence of their width is neglected. (In a local description, the width of the Hubbard bands is approximately given by $W$ 1].) In Fig. 3 we show that this approximation indeed has only a minor effect on the electronic properties of the metallic wide band. Keeping $W_{2}=4 \mathrm{eV}$ fixed and changing $W_{1}$ between $0.1 W_{2}$ and $0.5 W_{2}$, we notice that, both for isotropic and anisotropic exchange coupling, the selfenergy of the wide band at $U=0.6 W_{2}=2.4 \mathrm{eV}$ is almost unaffected by the original single-particle width of the insulating narrow band. The detailed electronic properties of these phases will be discussed in Sections 5 and 6 .
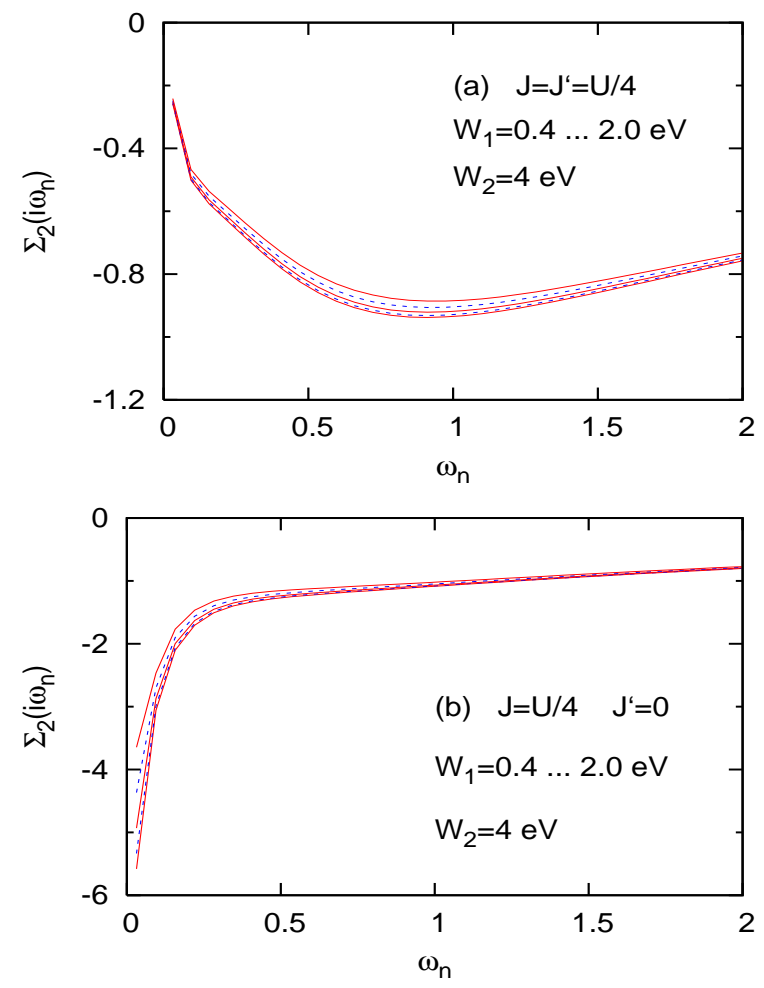

FIG. 3: Imaginary part of self-energy of wide band in intermediate phase for decreasing widths $W_{1}$ of narrow band, with fixed $W_{2}=4 \mathrm{eV}, U=2.4 \mathrm{eV}, n_{s}=8, T=10 \mathrm{meV}$. Alternating solid (red) and dashed (blue) curves: $W_{1}=$ $0.4,0.8,1.2,1.6,2.0 \mathrm{eV}$ (from bottom). (a) $J=J^{\prime}=U / 4$; (b) $J=U / 4, J^{\prime}=0$.

\section{NUMERICAL RENORMALIZATION GROUP APPROACH FOR EFFECTIVE ONE-BAND MODEL}

In the limit of an insulating narrow band interacting with a metallic wide band, the two-band model can be simplified to an effective one-band model by eliminating the high energy states associated with the upper and lower Hubbard bands of the narrow band 21]. Neglecting the one-electron hopping in the narrow band, i.e., assuming $W_{1}=0$, and fixing the occupations of these orbitals, the terms in Eq. (1) involving interorbital Coulomb and pair-exchange interactions disappear. The effective Hamiltonian then reduces to

$$
\begin{aligned}
H= & \sum_{l m \sigma} t_{l m} c_{l \sigma}^{+} c_{m \sigma}+U \sum_{l} n_{l \uparrow} n_{l \downarrow} \\
& -\sum_{l}\left[2 J S_{l}^{z} s_{l}^{z}+2 J^{\prime}\left(S_{l}^{+} s_{l}^{-}+S_{l}^{-} s_{l}^{+}\right)\right] .
\end{aligned}
$$

In this model, the low energy degrees of freedom of the narrow band are represented by local moments $\vec{S}_{l}$. These interact ferromagnetically with the local spin density $\vec{s}_{l}$ of the wide-band conduction electrons which are also subject to a local Coulomb repulsion $U$. The model corre- 
sponds to a double-exchange model with Coulomb interactions amongst the itinerant electrons or, equivalently, to the ferromagnetic Kondo lattice model with interactions in the band. The antiferromagnetic case, $J<0$, for $U=0$, has already been investigated in the context of heavy fermions [27]. We adapt these calculations to the case of interest here, namely, ferromagnetic exchange, $J>0$, and $U>0$. The equivalence between the full twoband model and the effective model holds provided $U$ is large enough so that a description of the low energy degrees of freedom of the narrow band in terms of local moments is possible and provided that $J^{\prime} \ll U / 2$. The latter condition corresponds to eliminated excited states of the full model being far from the first excited state of the effective model. Unless $J$ is chosen to be very small, we see that for realistic values of $J \approx U / 4$, the equivalence will hold well for Ising exchange coupling $\left(J^{\prime}=0\right)$ but less well for isotropic Hund's coupling $\left(J^{\prime}=J\right)$. In either case, the effective model should be increasingly accurate in the limit $\omega, T \rightarrow 0$.

Within DMFT, we need to solve an effective quantum impurity model corresponding to a $S=1 / 2$ Kondo impurity coupled ferromagnetically with conduction electrons subject to a local Coulomb repulsion:

$$
\begin{aligned}
H= & \sum_{k, \sigma} \varepsilon_{k} c_{k \sigma}^{+} c_{k \sigma}+U n_{0 \uparrow} n_{0 \downarrow} \\
& -2 J S_{0}^{z} s_{0}^{z}-2 J^{\prime}\left(S_{0}^{+} s_{0}^{-}+S_{0}^{-} s_{0}^{+}\right) .
\end{aligned}
$$

We solve this model using the numerical renormalization group method [28], which allows calculation of dynamical quantities on the real energy axis at both zero and finite temperature 29, 30]. The impurity self-energy $\Sigma(\omega)$ is calculated using the method described in Ref. 33]. For comparison with ED, it is then evaluated on the imaginary axis $z=i \omega$ by analytic continuation

$$
\Sigma(i \omega)=-\frac{1}{\pi} \int_{-\infty}^{+\infty} d \omega^{\prime} \frac{\operatorname{Im} \Sigma\left(\omega^{\prime}\right)}{i \omega-\omega^{\prime}} .
$$

The calculations use a logarithmic discretization of the conduction band $\varepsilon_{k_{n}} \rightarrow \pm D_{2} \Lambda^{-\frac{n-1}{2}}$ with $\Lambda=1.5$ and we retain of order 600 states per NRG iteration. Details of the calculation of spectra and other dynamical quantities can be found in Refs. 29, 34].

The effective model specified in Eq. (3) allows the nonFermi-liquid physics of the intermediate phase of the twoband model to be understood qualitatively 21. The case of Ising exchange $\left(J^{\prime}=0\right)$ describes interacting conduction electrons scattering from a disordered static configuration of local spins with $S_{l}^{z}= \pm 1 / 2$. The disorder potential is proportional to the Ising exchange $J$. The conduction electrons therefore have a finite lifetime at the Fermi level even at $T=0$, as is characteristic of a disordered metal. Switching on the spin-flip part of the Hund's exchange gives the disordered spin configuration some dynamics at finite temperature, but as long as
$J^{\prime}<J$, we expect from the ferromagnetic Kondo model that the spin-flip part of the Hund's exchange will renormalize to zero at low temperature with the Ising part of the Hund's exchange remaining finite. At $T=0$, the system will again be disordered with a disorder potential given by the finite Ising part of the Hund's exchange. This results in a finite lifetime for the conduction electrons at $T=0$.

The situation changes for isotropic Hund's exchange $\left(J^{\prime}=J\right)$. In this case, the ferromagnetic exchange in the effective impurity model (4) is known to be marginally irrelevant [31, 32]. Thus, both Ising and spin-flip parts of the Hund's exchange renormalize to zero and there will be no disorder scattering at $T=0$, giving rise to an infinite lifetime for the conduction electrons at $T=0$. The vanishing of the self-energy at $\omega=T=0$ also implies that the narrow-band spectral function satisfies a pinning condition at $\omega=T=0$. In Sections 5 and 6 , these qualitative considerations will be seen to be in accord with the numerical results from ED for the full two-band model and NRG results for the effective one-band model.

\section{METALLIC PHASE}

We begin our discussion of the multi-band ED results with the region in which both subbands are metallic, just below the Mott transition associated with the narrow band. To illustrate the applicability of the ED approach this region is very useful since it involves important redistribution of spectral weight between low and high frequencies that must be captured properly in order to describe the Mott transition.

Fig. 4 . shows a comparison of the subband self-energies for $n_{s}=8$ and $n_{s}=6$ in the region close to the lower critical Coulomb energy for $T=2.5 \mathrm{meV}$. For $n_{s}=8$, the $\Sigma_{i}\left(i \omega_{n}\right)$ vary roughly linearly at low frequencies, indicating that both bands are metallic. Of course, the narrow band is more strongly correlated than the wide band. The spacing between the lowest excited state and the ground state energy in these calculations is typically $1-2 \mathrm{meV}$, i.e., evaluation of the self-energies in this low temperature range is indeed meaningful.

The results for $n_{s}=6$ are similar, except that the self-energy of the narrow band at $U=2.1 \mathrm{eV}$ is inversely proportional to $i \omega_{n}$, indicating that the Mott transition in this case is located between $U=2.0 \mathrm{eV}$ and $U=2.1 \mathrm{eV}$. In addition, at the lowest Matsubara frequencies the self-energy of the wide band at $2.1 \mathrm{eV}$ exhibits deviations from linear $i \omega_{n}$ variation. These deviations are related to finite-size effects and will be analyzed in more detail in the next section when we discuss the intermediate orbital-selective phase. Just below the transition for $n_{s}=6$, at $U=2 \mathrm{eV}$, very small deviations from approximately linear $i \omega_{n}$ variation can also be seen in both $\Sigma_{i}\left(i \omega_{n}\right)$. 

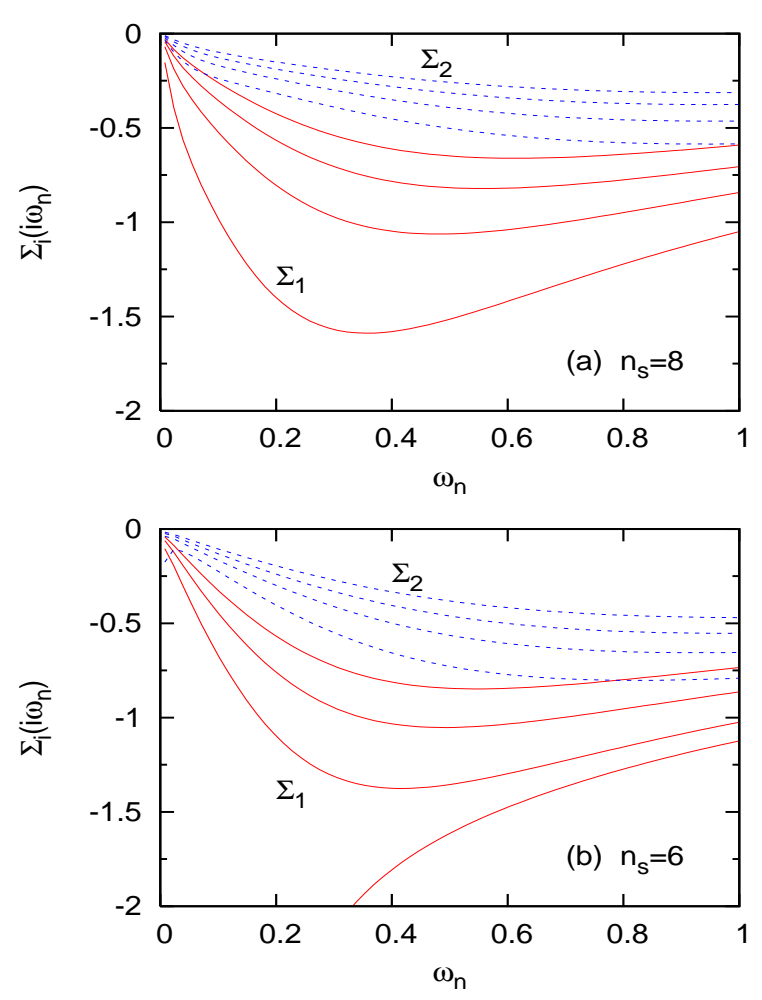

FIG. 4: Imaginary part of subband self-energies in metallic region for $J=J^{\prime}=U / 4$ at $T=2.5 \mathrm{meV}$. Solid (red) curves: narrow band, dashed (blue) curves: wide band. From top: $U=1.8,1.9,2.0,2.1 \mathrm{eV}$. (a) $n_{s}=8$; (b) $n_{s}=6$.

It is remarkable that the ED results for $n_{s}=8$ capture the correlation effects in the common metallic phase of both subbands very well until close to $U_{c 1}$ and down to rather low temperatures. This result is not at all obvious since so close to the Mott transition a large fraction of the spectral weight of the narrow band is transfered from the Fermi level to the Hubbard bands. Even the results for $n_{s}=6$ are qualitatively correct. The main effect of the cluster size $n_{s}=6$ is the underestimate of $U_{c 1}$ by about $0.1 \mathrm{eV}$. According to the single-band results [16] inclusion of the zero energy bath levels in the $n_{s}=8$ cluster should provide the most important part of the shift towards the correct $U_{c 1}$. In addition these extra bath levels yield an excellent representation of the metallic properties of both subbands right up to the critical Coulomb energy.

Of course, slight finite-size effects should be manifest also in the low-temperature ED results for $n_{s}=8$. The precise form of $\Sigma_{i}\left(i \omega_{n}\right)$ at very low frequencies and temperatures, in particular, the range of true Fermi-liquid behavior in the immediate vicinity of $U_{c 1}$, can only be investigated by more accurate methods, such as a full two-band extension of the NRG approach which is applicable at zero and finite $T$ [35].

We emphasize that this region of the $T / U$ phase diagram for isotropic Hund's exchange is not yet accessi-
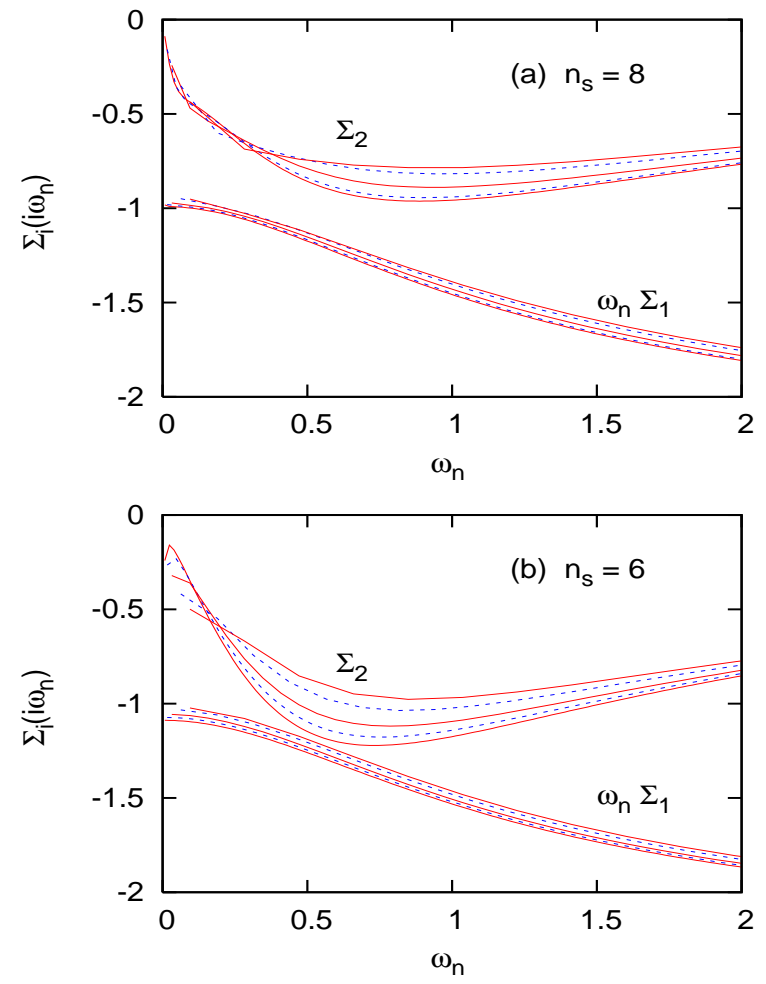

FIG. 5: Imaginary part of subband self-energies in intermediate phase, for $U=2.4 \mathrm{eV}$ and $J=J^{\prime}=U / 4$. $T=2.5,5,10,20,30 \mathrm{meV}$ (from bottom). (a) $n_{s}=8$; (b) $n_{s}=6$;

ble using QMC calculations. It would therefore be of great interest to extend the present results to $n_{s}=9$ and $n_{s}=12$ in order to explore the strongly correlated metallic phase of realistic three-band materials which have so far been investigated only for Ising-like exchange interactions. A more detailed comparison of the role of Hund vs. Ising exchange treatments in the metallic phase of the present two-band model will be given elsewhere [36].

\section{INTERMEDIATE PHASE: ISOTROPIC HUND'S EXCHANGE}

As shown first by Koga et al. 12] within ED/DMFT calculations at $T=0$, the two-band Hubbard model with full Hund's exchange interaction exhibits successive, orbital-selective Mott transitions. In Ref. [16] we proved that analogous ED calculations at finite temperature are consistent with these findings, revealing sequential firstorder transitions associated with the two subbands. In this section we discuss in more detail the low temperature properties of the intermediate phase between the fully metallic and insulating phases.

Fig. 15 shows the subband self-energies for a Coulomb energy $U=2.4 \mathrm{eV}$, i.e., between the lower and upper Mott transitions. The results for $n_{s}=8$ demonstrate 


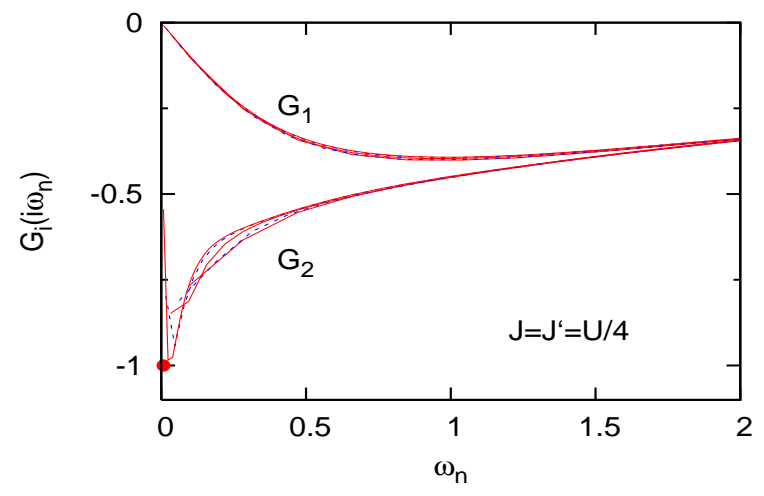

FIG. 6: Imaginary part of subband Green's functions in intermediate phase, for $U=2.4 \mathrm{eV}, J=J^{\prime}=U / 4, n_{s}=8$. $T=2.5,5,10,20,30 \mathrm{meV}$. The dot indicates the pinning condition for the wide band.

that the narrow band is insulating, i.e., its self-energy is inversely proportional to $i \omega_{n}$ at low frequencies. $\Sigma_{1}\left(i \omega_{n}\right)$ is also nearly independent of temperature in the range $T=2.5 \ldots 30 \mathrm{meV}$, suggesting that the Mott gap is considerably larger. The self-energy of the wide band, however, extrapolates to zero at low frequencies, indicating metallic behavior. These results suggest that the pinning condition $N_{2}(0)=A_{2}(0)$ is satisfied, i.e., the spectral density at $E_{F}$ of the interacting system coincides with that of the non-interacting system. Because of the rapid increase of $\operatorname{Im} \Sigma_{2}\left(i \omega_{n}\right)$ at small $\omega_{n}$, the spectral distribution of the wide band in the intermediate phase consists of a very narrow peak at $E_{F}$ and large upper and lower Hubbard bands. Nevertheless, as will be discussed further below, the comparison with the NRG results indicates that this band does not satisfy true Fermi-liquid behavior.

The results for $n_{s}=6$ shown in Fig. [5) agree qualitatively with those for $n_{s}=8$. The self-energies of the narrow band are nearly identical, with only a slightly larger dependence on temperature in the case $n_{s}=6$. The main difference is that $\Sigma_{2}\left(i \omega_{n}\right)$ at low $T$ and small $i \omega_{n}$ exhibits larger deviations as a result of finite-size effects. This is plausible since, as discussed above, the wide band is metallic, but highly correlated. The pronounced three-peak structure of its spectral function is therefore not represented accurately by only two non-zero bath levels per impurity orbital in the ED calculation for $n_{s}=6$. The extra bath level with zero energy included for $n_{s}=8$ therefore plays a crucial role for a proper description of the metallic behavior of the wide band for full Hund's coupling.

Fig. [6] shows the subband Greens functions $G_{i}\left(i \omega_{n}\right)$ in the intermediate phase at various temperatures. $\operatorname{Im} G_{1}$ is linear in $\omega_{n}$ at low frequencies, as expected for the insulating subband. Although the wide band exhibits clear signs of finite-size effects at small $\omega_{n}$ it is nevertheless seen to approximately satisfy the pinning condition
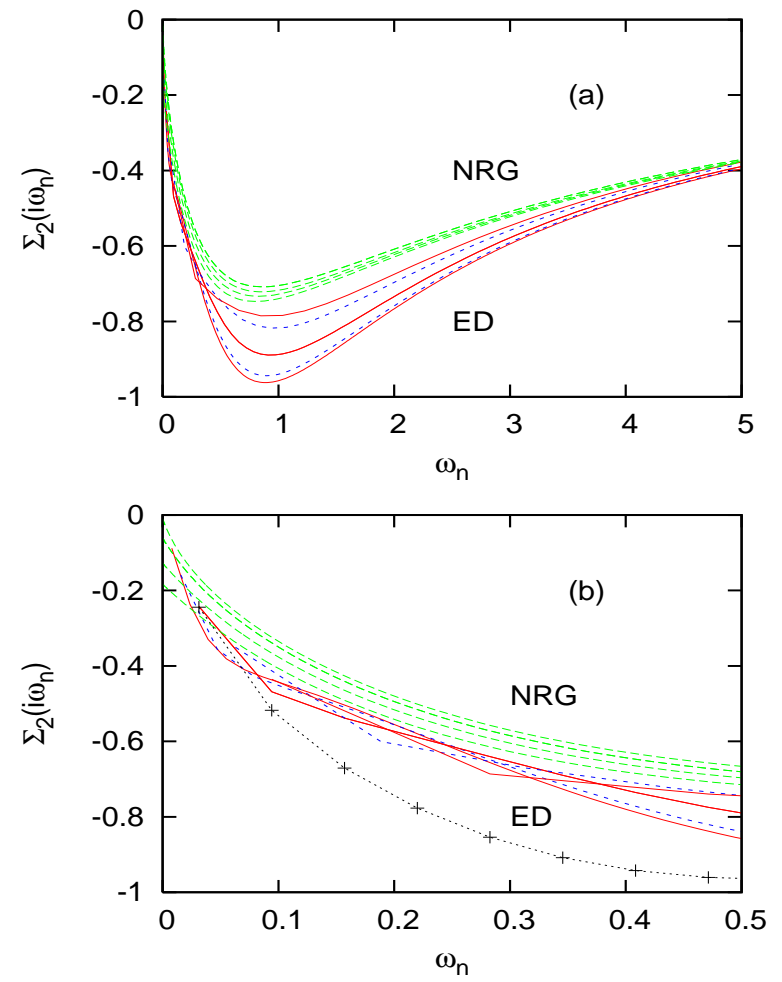

FIG. 7: Imaginary part of self-energy $\Sigma_{2}$ of wide band for $U=2.4 \mathrm{eV}$ and $J=J^{\prime}=U / 4$. (a) Solid (red) and dashed (blue) curves: ED results for $n_{s}=8$ at $T=$ 2.5, 5, 10, 20, $30 \mathrm{meV}$ (from bottom); green curves: NRG results for $T=3,10,15,34 \mathrm{meV}$ (from top). (b) Same quantities on expanded low-frequency scale. Black curve $(+)$ : self-energy $\Sigma_{1}$ of narrow band at $2.0 \mathrm{eV}$.

$\operatorname{Im} G_{2}\left(i \omega_{n}\right) \rightarrow-\pi N_{2}(0)=-1$ in the limit $\omega_{n} \rightarrow 0$.

For computational reasons the role of finite-size effects in the present finite-temperature ED approach based on full matrix diagonalization can at present not be checked by going beyond the cluster size $n_{s}=8$. To analyze the low-frequency properties of the wide band in the intermediate phase more closely, we have carried out NRG calculations for the effective one-band model discussed in Section 3. Although this model is more appropriate for the anisotropic exchange treatment discussed in the next section, we use it here (with some caution) since it is so far the only scheme capable of providing a guideline for the two-band Hubbard model at low finite temperatures.

In Fig. [7(a) the ED results for $\operatorname{Im} \Sigma_{2}\left(i \omega_{n}\right)$ are compared with the corresponding self-energy derived within the NRG. The overall frequency variation of $\Sigma_{2}$ is seen to be remarkably similar for both methods, implying similar spectral distributions. The most noticeable difference is the larger dependence on temperature in the case of the ED results. According to Fig. 5 the increase in cluster size from $n_{s}=6$ to $n_{s}=8$ diminishes the variation of $\Sigma_{2}$ with temperature and makes the minimum near $\omega_{n} \approx 0.9$ less deep. This trend indicates that larger cluster sizes 


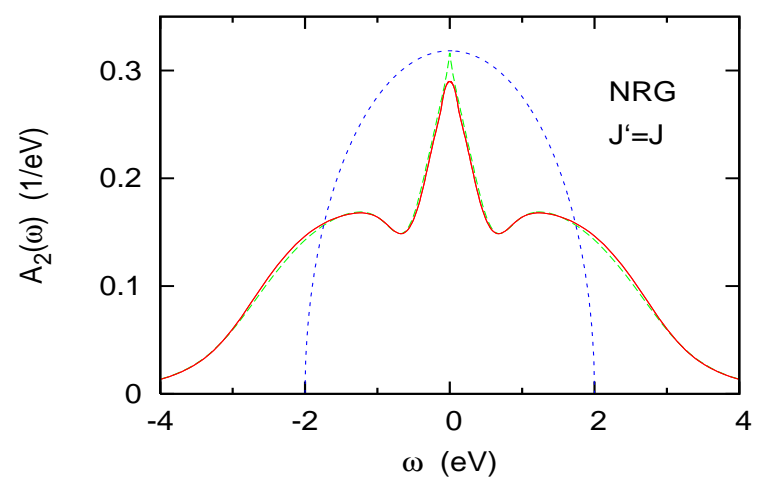

FIG. 8: Spectral distribution of wide band in intermediate phase, calculated within NRG approach for $U=2.4 \mathrm{eV}, J=$ $J^{\prime}=U / 4$. Green curve: $T=3 \mathrm{meV}$; red curve: $T=30 \mathrm{meV}$; dashed blue curve: bare density of states.

would bring the ED results into better agreement with the NRG data. We point out, however, that part of the difference should be caused by the approximate nature of the NRG model in the case $J^{\prime}=J$. As shown in the following section, for $J^{\prime}=0$ the ED and NRG results for $\Sigma_{2}$ at $T=20 \ldots 30 \mathrm{meV}$ are in perfect agreement.

The low-frequency region of $\Sigma_{2}\left(i \omega_{n}\right)$ is plotted in greater detail in Fig. 7(b). In the ED calculation, the spacing between the lowest excited level and the ground state energy is typically $10 \mathrm{meV}$. Thus, temperatures below this range will give rise to increasing finite-size effects, precluding any meaningful analysis at low frequencies. Despite this limitation, the ED data follow the frequency dependence of the NRG results rather well.

In order to provide some insight into the variation of $\Sigma_{2}$ at finite $\omega_{n}$, we compare it in Fig. (7) b) also with the self-energy of the narrow band $\Sigma_{1}$ at $U=2.0 \mathrm{eV}$, i.e., just below the Mott transition at $U_{c 1} \approx 2.1 \mathrm{eV}$. According to Fig. 1 (a), we find: $Z_{1}(U=2.0 \mathrm{eV}) \approx Z_{2}(U=2.4 \mathrm{eV}) \approx$ 0.11 . Nevertheless, whereas $\Sigma_{1}$ at $U=2.0 \mathrm{eV}$ exhibits the typical behavior expected for a strongly correlated Fermi-liquid phase, $\Sigma_{2}$ evidently has much larger nonlinear corrections. A similar systematic difference is found between $\Sigma_{2}$ in the intermediate phase and the behavior the self-energy in a single-band model for $U$ just below the Mott transition.

The NRG results shown in Fig. 7 indicate that $\Sigma_{2}\left(i \omega_{n}\right) \rightarrow 0$ in the limit $\omega_{n} \rightarrow 0$ and for low $T$. Thus, particles at $E_{F}$ have infinite lifetime. In addition, analogous NRG calculations at $T=0$ yield [35] non-Fermiliquid behavior, in agreement with Ref. 21]. Thus, at small finite $i \omega_{n} \operatorname{Im} \Sigma_{2}$ does not vary linearly. The qualitative agreement between the finite- $T$ NRG and ED results seen in Fig. [7 shows that the ED data are consistent with non-Fermi-liquid behavior.

Fig. 8 shows the spectral function of the wide band in the intermediate phase, calculated within the NRG.
Spectra of this kind were also obtained by Arita and Held [5] for $U$ near $U_{c 1}$ within QMC/DMFT calculations for the same two-band model for $T=0$ and $J^{\prime}=J=U / 4$. As mentioned above, the distribution exhibits a very narrow peak which satisfies the pinning condition at $E_{F}$ for temperatures up to about $10 \mathrm{meV}$. Because of the sharp central peak of $A_{2}(\omega)$, the real and imaginary parts of the self-energy of the wide band $\Sigma_{2}(\omega)$ exhibit structure on a similar scale. Nevertheless, the variation of $\operatorname{Im} \Sigma_{2}\left(i \omega_{n}\right)$ obtained in the NRG is very smooth. Thus, the weak shoulder seen in the ED results for $\Sigma_{2}$ near $\omega_{n} \approx 0.06$ in Figs. [3(a), [a) and 7 must be attributed to finite-size effects.

The ED and NRG results discussed above demonstrate that, in the presence of full Hund's exchange, the wide band above the main Mott transition at $U_{c 1}$ remains metallic, without satisfying Fermi-liquid criteria. In the following section it will be shown that the absence of spin-flip and pair-exchange terms enhances this trend towards non-Fermi-liquid behavior, so that even particles at $E_{F}$ acquire a finite lifetime. Thus, in both cases, the intermediate phase is bad-metallic.

We point out that the differences between the selfenergies for $n_{s}=8$ and $n_{s}=6$ appear mainly at rather low temperatures, below about $T=20 \mathrm{meV}$. Since QMC calculations also become difficult to converge in this range, three-band ED calculations with two bath levels per impurity orbital, i.e., cluster size $n_{s}=9$, should be competitive with analogous QMC calculations - with the important additional benefit, that the ED approach can handle full Hund's coupling.

\section{INTERMEDIATE PHASE: ISING EXCHANGE}

As shown in Ref. 13], in the absence of spin-flip and pair-exchange contributions, the first-order Mott transition at $U_{c 1} \approx 2.1 \mathrm{eV}$ affects the different subbands of the Hubbard model in fundamentally different ways: The narrow band undergoes a complete metal insulator transition, but the wide band changes from a normal metal to a bad metal in the sense that its self-energy exhibits progressive deviations from Fermi-liquid linear $\omega_{n}$ variation at low frequencies. Thus, $\operatorname{Im} \Sigma_{2}\left(i \omega_{n}\right) \rightarrow c(U)$ for $\omega_{n} \rightarrow 0$, where $c(U) \approx 0 \rightarrow-\infty$ for $U=U_{c 1} \rightarrow U_{c 2}$. Spectral functions obtained via the maximum entropy method showed that this breakdown of Fermi-liquid behavior in the wide band in the intermediate phase leads to a narrow pseudogap near $E_{F}$ and to a violation of the pinning condition, i.e., $A_{2}(0)<N_{2}(0)$. The spectral function of the wide band then acquires a characteristic four-peak structure, with two maxima flanking the pseudogap, in addition to the Hubbard bands at higher energies. With increasing $U$, the pseudogap becomes deeper and wider, until it turns into a true insulating gap at $U_{c 2} \approx 2.7 \mathrm{eV}$. As also shown in Ref. 13], the upper bad-metal to insu- 

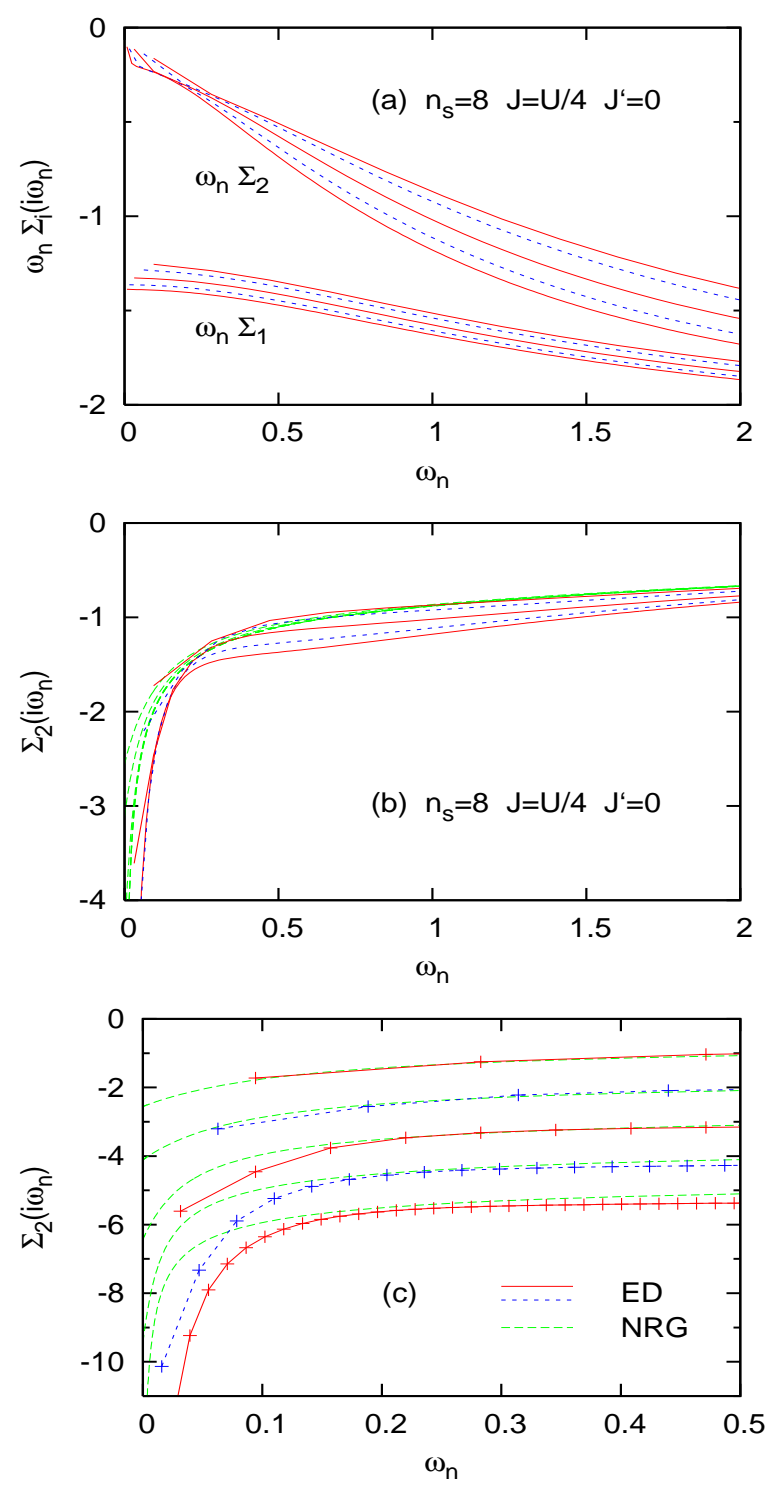

FIG. 9: Imaginary part of subband self-energies $\Sigma_{i}\left(i \omega_{n}\right)$ in intermediate phase for $n_{s}=8, U=2.4 \mathrm{eV}, J=U / 4$, $J^{\prime}=0$; Solid (red) and dashed (blue) curves: ED results for $T=2.5,5,10,20,30 \mathrm{meV}$ (from bottom). (a) $\omega_{n} \Sigma_{i}\left(i \omega_{n}\right)$; (b) $\Sigma_{2}\left(i \omega_{n}\right)$; green curves: NRG results as in (c). (c) Lowfrequency behavior of self-energy of wide band: red and blue curves: ED results as in (b); green curves: NRG results for $T=2,4.4,10,20,30 \mathrm{meV}$ (from bottom). For clarity, successive curves in (c) are displaced vertically by 1 .

lator transition is not a first-order transition, in contrast to the main transition at $U_{c 1}$.

In this section we discuss the temperature dependence of the subband self-energies in the intermediate phase and the finite-size effects associated with the limited number of bath levels included in our ED/DMFT approach.

Fig. 9 shows the subband self-energies for $n_{s}=8$ and $U=2.4 \mathrm{eV}$, assuming again $J=U / 4$, but $J^{\prime}=0$. The
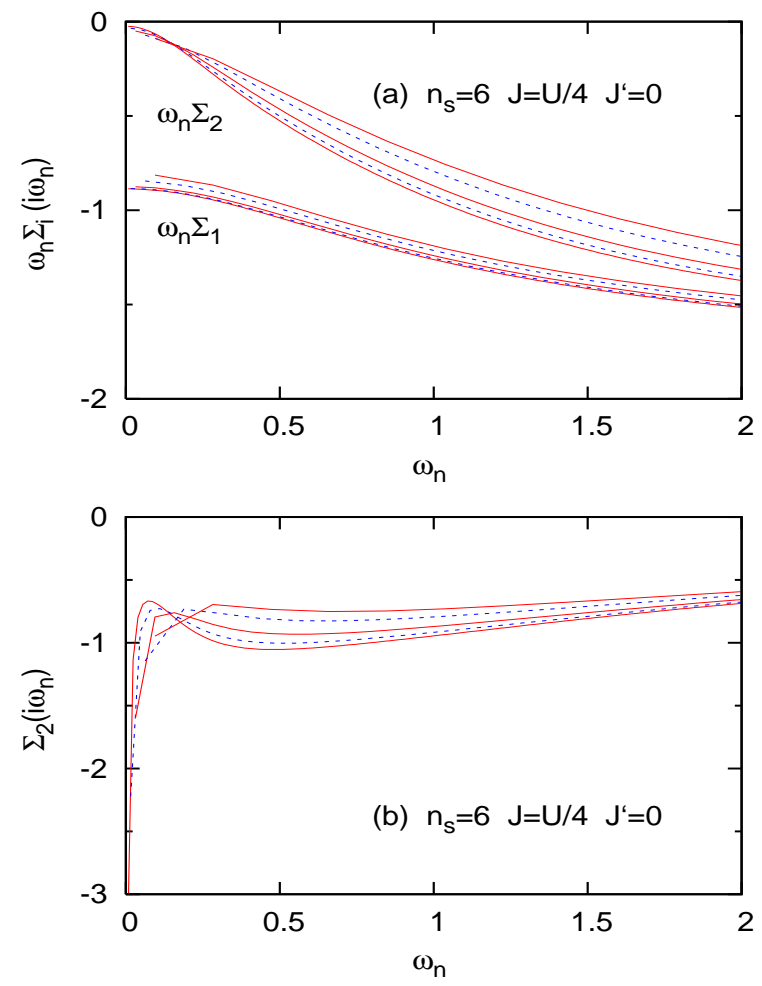

FIG. 10: Imaginary part of subband self-energies $\Sigma_{i}\left(i \omega_{n}\right)$ in intermediate phase for $n_{s}=6, U=2.2 \mathrm{eV}$ and $J=U / 4$, $J^{\prime}=0 . T=2.5,5,10,20,30 \mathrm{meV}$ (from bottom).

narrow band is fully insulating, so that $\omega_{n} \operatorname{Im} \Sigma_{1}\left(i \omega_{n}\right) \rightarrow$ const. nearly independently of temperature, similarly to

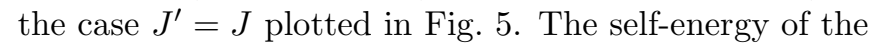
wide band, however, differs qualitatively from the behavior found for isotropic Hund's coupling. Rather than vanishing in the limit $\omega_{n} \rightarrow 0, \operatorname{Im} \Sigma_{2}\left(i \omega_{n}\right)$ now approaches a sharp minimum, which gets more pronounced towards low temperatures. Clearly, since $\omega_{n} \operatorname{Im} \Sigma_{2}\left(i \omega_{n}\right) \rightarrow 0$ at small $\omega_{n}$, this band is not yet insulating. Thus, instead of satisfying the pinning condition at $E_{F}$, the spectral function of the wide band exhibits a dip or pseudogap at the Fermi level (see below).

Fig. 9(b) also shows the NRG results for $\Sigma_{2}\left(i \omega_{n}\right)$. They are seen to be in very good agreement with the ED data, except at low frequencies for $T \leq 10 \mathrm{meV}$. The fact that for $T=20 \ldots 30 \mathrm{meV}$ there is now much better coincidence between the ED and NRG results than in Fig. 7 for $J^{\prime}=J$ indicates that, as discussed in Section 3 , the effective one-band model employed for the NRG is more appropriate in the case $J^{\prime}=0$. The lowfrequency behavior of $\operatorname{Im} \Sigma_{2}\left(i \omega_{n}\right)$ is shown in more detail in Fig. 9(c). Both the ED and NRG results reveal that the sharp minimum of $\Sigma_{2}$ at small $\omega_{n}$ gets progressively deeper at low temperatures, suggesting that the pseudogap in the spectral function becomes accordingly deeper.

Analogous results for $n_{s}=6$ are given in Fig. [10] Since 


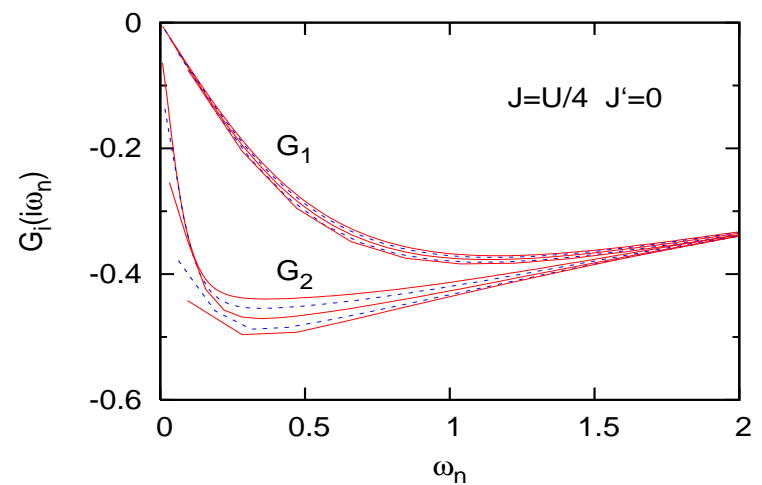

FIG. 11: Imaginary part of subband Green's functions in intermediate phase, for $U=2.4 \mathrm{eV}, J=U / 4, J^{\prime}=0, n_{s}=8$. $T=2.5,5,10,20,30 \mathrm{meV}$ (from top).

according to Ref. 16] in this case the wide band becomes insulating near $U \approx 2.4 \mathrm{eV}$, we choose $U=2.2 \mathrm{eV}$ to illustrate the non-Fermi-liquid behavior of this band in the intermediate phase. As for $n_{s}=8$, the narrow band is insulating, i.e., $\omega_{n} \operatorname{Im} \Sigma_{1}\left(i \omega_{n}\right) \rightarrow$ const. at low frequencies. The frequency variation of $\Sigma_{2}\left(i \omega_{n}\right)$ is seen to be more strongly affected by finite-size effects. Nevertheless, as in the case $n_{s}=8$, a sharp minimum is found for $\omega_{n} \rightarrow 0$.

Fig. 11] shows the subband Green's functions $G_{i}\left(i \omega_{n}\right)$ for anisotropic Hund's exchange at various temperatures. As for the case $J^{\prime}=J$ shown in Fig. 6] $\operatorname{Im} G_{1}$ is linear in $\omega_{n}$ at low frequencies, since the narrow band is insulating. On the other hand, because of the more severe breakdown of Fermi-liquid behavior in the wide band for $J^{\prime}=0$, with $\operatorname{Im} \Sigma_{2}\left(i \omega_{n}\right) \neq 0$ in the low-frequency limit, the Green's function no longer satisfies the pinning condition. Thus, $\operatorname{Im} G_{2}\left(i \omega_{n}\right) \rightarrow-c(T)$ with $c(T)<\pi N_{2}(0)=1$, and $c(T) \rightarrow 0$ for decreasing temperature. This behavior implies that the spectral function of the wide band exhibits a pseudogap at $E_{F}$ which becomes progressively deeper towards low $T$.

This picture is fully confirmed by the NRG results shown in Fig. 12 In contrast to the narrow peak at $E_{F}$ in the three-peak structure seen in Fig. 8 for isotropic Hund's coupling, the spectra for $J^{\prime}=0$ show a pseudogap which becomes deeper as $T$ decreases. As a result, the spectral distribution now exhibits a characteristic four-peak structure, with two maxima limiting the pseudogap and two shoulders associated with the Hubbard peaks. Spectra of this kind were first observed in the QMC/DMFT results at $T=31 \mathrm{meV}$ reported in Ref. 13] (see also next section). The comparison with the spectra for $J^{\prime}=J$ reveals nearly identical excitations for $|\omega| \gtrsim J$. Thus, as expected, the different treatments of exchange interactions affect primarily the low-frequency excitations in the metallic wide band.

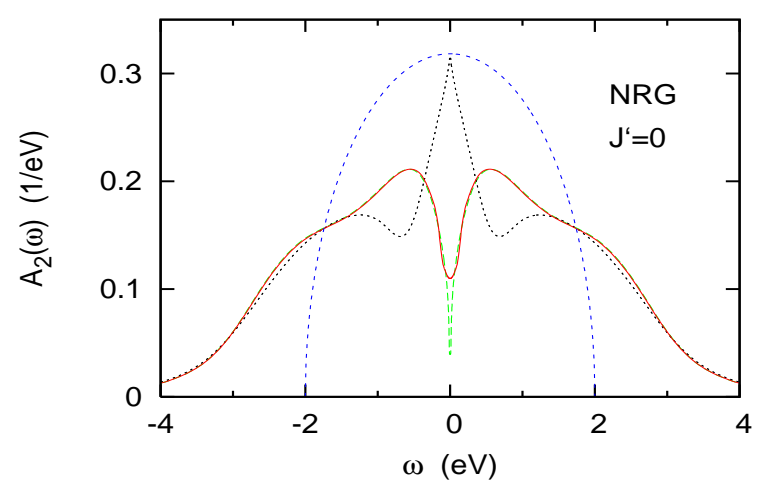

FIG. 12: Spectral distribution of wide band in intermediate phase, calculated within NRG approach for $U=2.4 \mathrm{eV}, J=$ $U / 4, J^{\prime}=0$. Green curve: $T=3 \mathrm{meV}$; red curve: $T=$ $34 \mathrm{meV}$; dashed blue curve: bare density of states. Black dotted curve: spectrum for $J^{\prime}=J, T=3 \mathrm{meV}$ from Fig. 8

\section{COMPARISON WITH PREVIOUS QMC/DMFT RESULTS}

In this and the following sections we compare the ED and NRG results for $J^{\prime}=0$ with available QMC/DMFT data in order to illustrate the consistency between these impurity treatments and to explore further the role of finite-size effects in the ED approach.

As shown in Ref. [13], in the absence of spin-flip and pair-exchange terms, purely metallic and insulating phases exist for $U<U_{c 1} \approx 2.1 \mathrm{eV}$ and $U>U_{c 2} \approx 2.7 \mathrm{eV}$, respectively. Fig. 13 shows a comparison of subband self-energies at three representative Coulomb energies within the intermediate 'bad-metal' non-Fermi-liquid region. The ED results for $n_{s}=8$ are seen to be in excellent agreement with the NRG self-energies, confirming the validity of the effective one-band model in the intermediate phase for $J^{\prime}=0$. Moreover, both schemes agree very well with the QMC/DMFT self-energies reported in Ref. 13 for $T=31 \mathrm{meV}$. Minor differences between the QMC and ED/NRG results are found only in the steepest parts of $\Sigma_{i}$ close to the first Matsubara frequency. (In contrast to the QMC calculations which are carried out at discrete Matsubara frequencies, the NRG self-energy is available continuously as a function of frequency. The ED self-energies could in principle also be obtained at arbitrary $i \omega$ but were calculated here at $i \omega_{n}$. This explains the slightly different form of the curves plotted in panel (c). At $i \omega_{n}$ the ED and NRG data nearly coincide.)

The overall trend obtained previously within the QMC/DMFT is fully confirmed by the new ED and NRG calculations: The narrow band is insulating throughout this range of Coulomb energies, whereas the wide band changes gradually from metallic to insulating via progressive non-Fermi-liquid behavior. Thus, for small $\omega_{n}$, $\operatorname{Im} \Sigma_{2}\left(i \omega_{n}\right) \rightarrow c(U)$, where $c(U) \rightarrow-\infty$ at $U \approx 2.7 \mathrm{eV}$. 

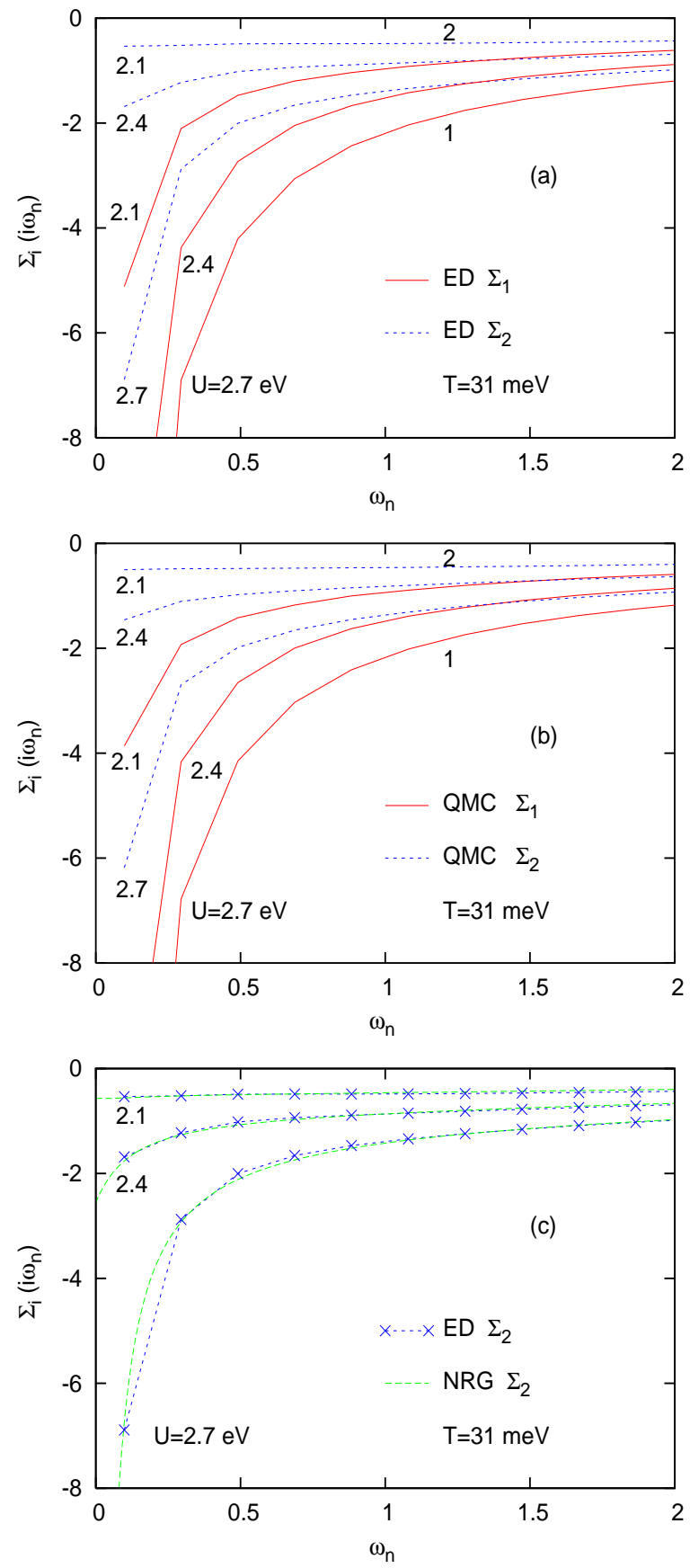

FIG. 13: Comparison of subband self-energies $\Sigma_{i}\left(i \omega_{n}\right)$ in intermediate non-Fermi-liquid phase for $U=2.1,2.4,2.7 \mathrm{eV}$, $J=U / 4, J^{\prime}=0, T=31 \mathrm{meV}$, calculated via three different impurity solvers: (a) ED results for $n_{s}=8$ : red solid curves: narrow band; blue dashed curves: wide band; (b) QMC results from Fig. 10 of Ref. [13]; red solid curves: narrow band; blue dashed curves: wide band; (c) comparison of ED (x) and NRG self-energies of wide band.

Evidently, despite their intrinsic numerical uncertainties, the three complementary impurity solvers: ED, NRG and QMC provide perfectly consistent descriptions of the electronic properties of the orbital-selective phase
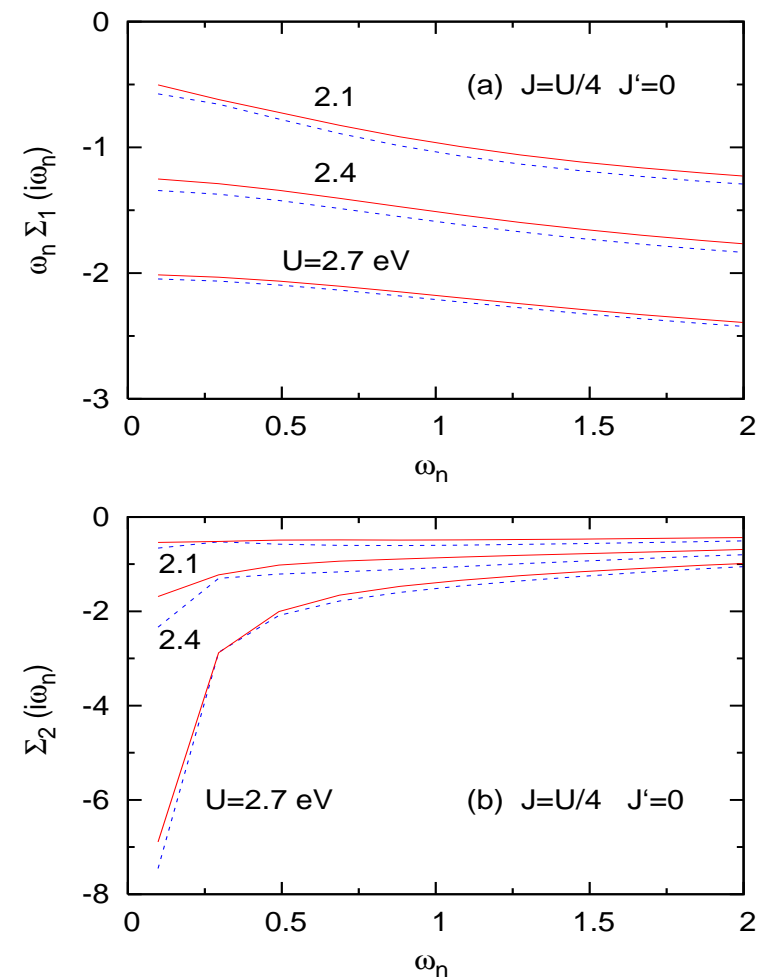

FIG. 14: Subband self-energies $\Sigma_{i}\left(i \omega_{n}\right)$ in intermediate nonFermi-liquid phase for $T=31 \mathrm{meV}, U=2.1,2.4,2.7 \mathrm{eV}$, $J=U / 4, J^{\prime}=0$. Solid (red) curves: $n_{s}=8$; dashed (blue) curves: $n_{s}=6$. (a) narrow subband: $\omega_{n} \Sigma_{1}\left(i \omega_{n}\right)$; (b) wide subband: $\Sigma_{2}\left(i \omega_{n}\right)$.

for Ising-like exchange.

Since the temperature $T=31 \mathrm{meV}$ in these results is relatively high, finite-size effects tend to be less pronounced than in the cases discussed in the previous sections at lower $T$. This is illustrated in Fig. 14 where we compare the above ED results for $n_{s}=8$ with those for $n_{s}=6$. The self-energies of the narrow band are nearly identical and satisfy $\omega_{n} \Sigma_{1}\left(i \omega_{n}\right) \rightarrow$ const. at low frequencies. This is plausible since the additional zero-energy bath level for $n_{s}=8$ carries very little weight in the insulating state. Because of breakdown of Fermi-liquid behavior, the self-energy of the wide band approaches a finite value in the limit $\omega_{n} \rightarrow 0$. The differences between the results for $n_{s}=6$ and $n_{s}=8$ are very small as long as this band is either nearly metallic, like at $U=2.1 \mathrm{eV}$, or nearly insulating, like at $U=2.7 \mathrm{eV}$. Slightly larger differences are found only in the middle of the bad-metallic region near $U=2.4 \mathrm{eV}$. As discussed in the preceding section, the spectral function then has a more complicated four-peak structure as a result of the narrow pseudogap at $E_{F}$, which cannot be adequately represented using only two bath levels. This region could possibly be even more accurately described by using four bath levels for the wide band and two levels for the insulating 


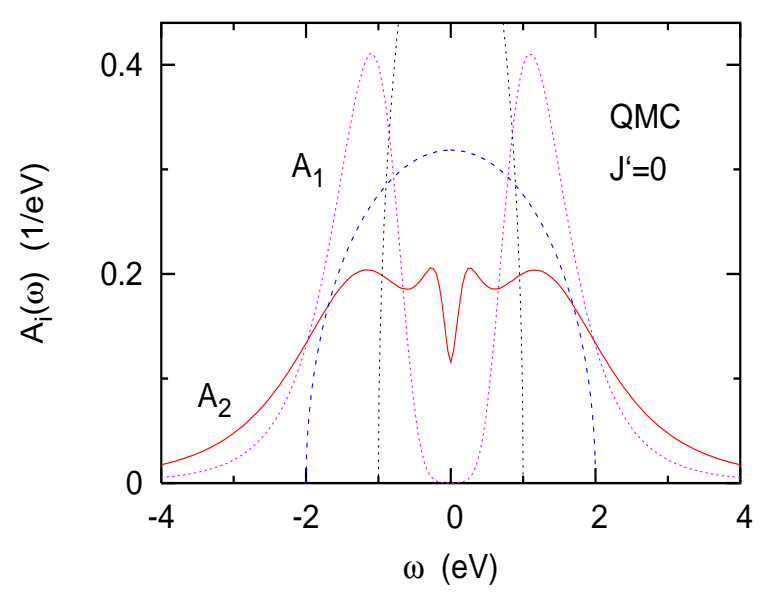

FIG. 15: Spectral distributions of subbands in intermediate phase, calculated within QMC/DMFT for $U=2.4 \mathrm{eV}$, $J=U / 4, J^{\prime}=0, T=31 \mathrm{meV}$. Red curve: metallic wide band; magenta curve: insulating narrow band; blue and black curves: bare densities of states. From Fig. 11(b) of Ref. [13].

narrow band, maintaining the total $n_{s}=8$.

We close this section by showing in Fig. [15] the spectral distributions of both subbands, as calculated within the QMC/DMFT and the maximum entropy method 13]. The spectrum of the bad-metallic wide band can be compared with the corresponding NRG spectra plotted in Fig. 12 Both distributions exhibit the marked four-peak structure induced by the pseudogap and the Hubbard bands. The low-frequency region is seen to be in excellent agreement. Both methods coincide in that, at $T \approx 30 \mathrm{meV}$, the interacting density of states at $E_{F}$ of the wide band is only about one third of the noninteracting one. The slightly larger differences at higher energies, in particular, the position and relative weight of the Hubbard bands, may be caused by the choice of fitting parameters in the maximum entropy procedure, and by the less accurate nature of the NRG approach at high energies.

\section{COMPARISON WITH QMC/DMFT RESULTS FOR $W_{2}=10 W_{1}$}

The breakdown of Fermi-liquid behavior in the orbitalselective phase with Ising-like exchange was recently also studied by Biermann et al. 21] for the same two-band Hubbard model as above, except for $W_{1}=0.2 \mathrm{eV}$ and $W_{2}=2 \mathrm{eV}$. The subbands were also assumed to be nonhybridizing and half-filled. QMC/DMFT calculations were carried out for $T=1 / 120 \ldots 1 / 40 \mathrm{eV}, U=0.8 \mathrm{eV}$, $J=0$ and $J=U / 4$, with $J^{\prime}=0$, i.e., in the absence of spin-flip and pair exchange terms. For $J=0$ as well as $J=U / 4$, the self-energy of the narrow band was found to diverge at low frequencies, demonstrating that this

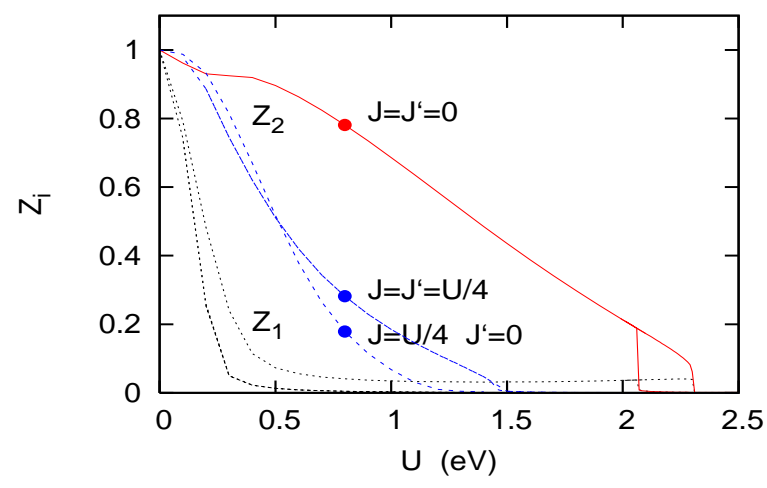

FIG. 16: $\quad Z_{i}(U)$ as a function of $U$ for $W_{1}=0.2 \mathrm{eV}, W_{2}=$ $2.0 \mathrm{eV}, T=1 / 120 \mathrm{eV}$, calculated within ED/DMFT, $n_{s}=6$, for $J=J^{\prime}=0, J=J^{\prime}=U / 4$, and $J=U / 4, J^{\prime}=0$. Red and blue curves: wide band, black curves: narrow band. The $Z_{1}$ for $J=J^{\prime}=U / 4$ and $J=U / 4, J^{\prime}=0$ are indistinguishable. The dots mark the three cases shown in Fig. [17]

band is insulating since $W_{1} \ll U$. The wide band is in the intermediate orbital-selective region between the purely metallic and insulating phases since $U \ll W_{2}$. The self-energy of this band showed a striking variation with the magnitude of $J$ : For $J=0, \Sigma_{2}\left(i \omega_{n}\right) \sim i \omega_{n}$ at low frequencies, as expected for Fermi-liquid behavior. For $J=U / 4$, however, $\operatorname{Im} \Sigma_{2}\left(i \omega_{n}\right) \rightarrow$ const. $\approx-0.09$ nearly independently of temperature for $T=1 / 120 \ldots 1 / 40 \mathrm{eV}$, indicating breakdown of Fermi-liquid properties. Accordingly, the pinning condition $N_{2}(0)=A_{2}(0)$ was found to be satisfied for $J=0$, but not for $J=U / 4$. These results are fully consistent with the trend discussed in Ref. 13. for $W_{1}=2 \mathrm{eV}$ and $W_{2}=4 \mathrm{eV}$.

To check the accuracy of our finite- $T$ ED approach we have applied it to the case investigated in Ref. 21]. To provide a picture of the Mott transitions in this two-band system we show first in Fig. 16] the variation of $Z_{i}(U)$ for three different treatments of Hund's exchange. In all cases the narrow band becomes insulating at about $U_{c 1}=0.3 \ldots 0.4 \mathrm{eV}$. However, the range and nature of the orbital-selective phase of the wide band, $U_{c 1}<U<U_{c 2}$, depend sensitively on the magnitudes of $J / U$ and $J^{\prime} / U$. For $J=J^{\prime}=0$ the upper transition occurs for $U_{c 2}$ slightly larger than $W_{2}$, with a pronounced hysteresis loop indicative of first-order behavior. A very weak hysteresis is found also for $J=J^{\prime}=U / 4$, with $U_{c 2} \approx 1.5 \mathrm{eV}$, similar to the one in Fig. 22 near $U \approx 3 \mathrm{eV}$. Finally, for $J=U / 4$ and $J^{\prime}=0, U_{c 2} \approx 1.2 \mathrm{eV}$ without evidence of first-order behavior. (The latter result is consistent with $U_{c 2} \approx 2.4 \mathrm{eV}$ in Fig. 2 of Ref. [16].) Although $Z_{2}(U) \approx 0.2 \ldots 0.8$ at $U=0.8 \mathrm{eV}$, i.e., for $U_{c 1} \ll U \ll U_{c 2}$, the analysis of the self-energy reveals fundamentally different electronic properties of the wide band in this region.

This is illustrated in Fig. 177 which shows $\operatorname{Im} \Sigma_{2}\left(i \omega_{n}\right)$ for $n_{s}=8$ and $n_{s}=6$. The ED results for $n_{s}=8$ 

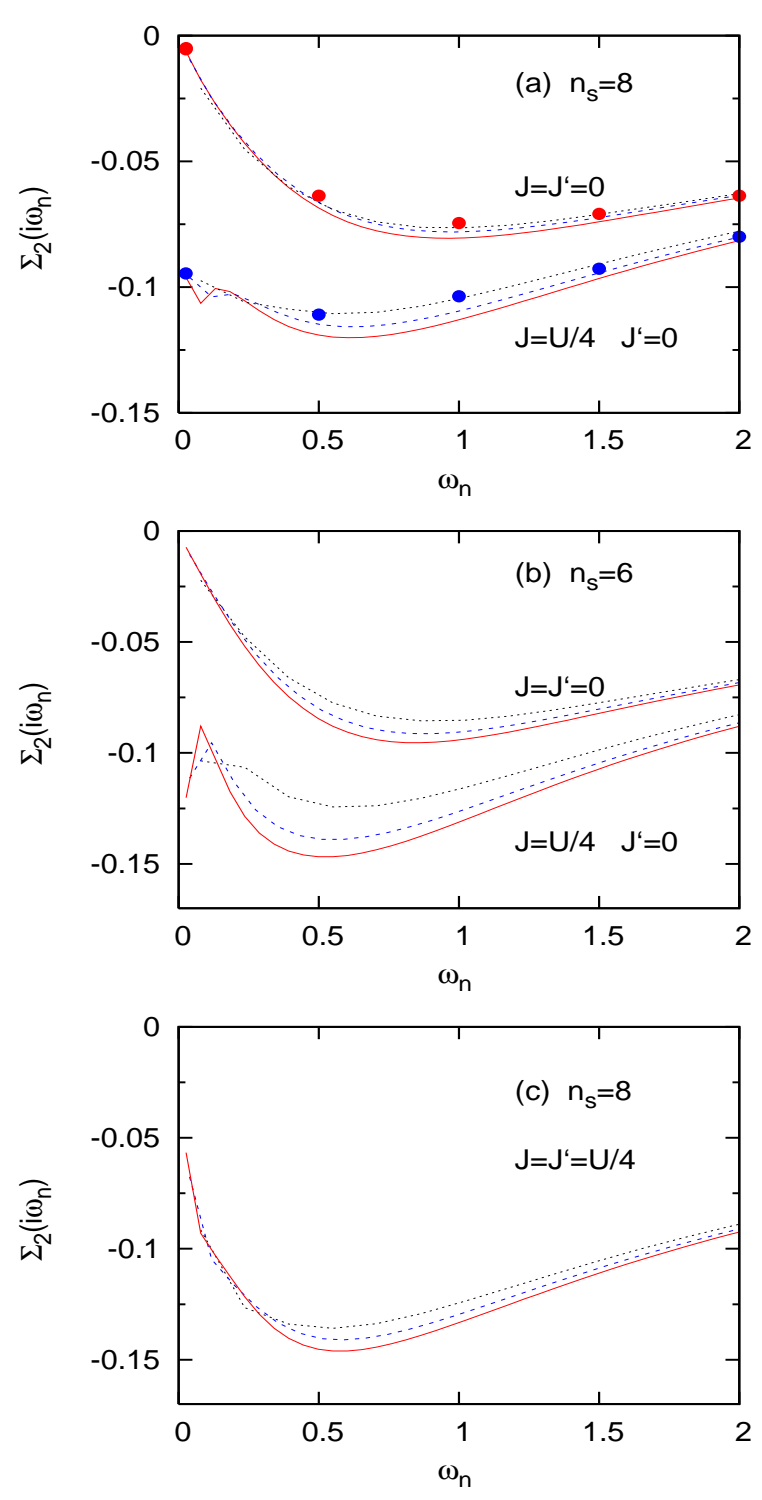

FIG. 17: (a) Imaginary part of self-energy of wide band for $J=0$ and $J=U / 4$ in intermediate phase for $U=0.8 \mathrm{eV}$, $J^{\prime}=0$, calculated within ED/DMFT with $n_{s}=8 . W_{1}=$ $0.2 \mathrm{eV}, W_{2}=2.0 \mathrm{eV}$. Red solid, blue dashed, and black dotted curves: $T=1 / 120 \mathrm{eV}, T=1 / 80 \mathrm{eV}$, and $T=1 / 40 \mathrm{eV}$, respectively. The dots denote the results for $T=1 / 120 \mathrm{eV}$ obtained in Ref. 21]. (b) Same as (a) except for $n_{s}=6$. (c) Same as (a) except for $J^{\prime}=J=U / 4$.

are in excellent agreement with the QMC data 21] for $J=J^{\prime}=0$ as well as $J=U / 4, J^{\prime}=0$, with the exception of small finite-size effects at the lowest Matsubara frequencies and lowest temperature. (Since $W_{2}=2 \mathrm{eV}$, $T=1 / 120 \ldots 1 / 40 \mathrm{eV}$ in these calculations corresponds to about $T=16 \ldots 50 \mathrm{meV}$ in the case $W_{2}=4 \mathrm{eV}$ considered in the preceding sections.) Also, the variation with $T$ is in our results slightly more pronounced than in the case of the QMC/DMFT.

For $n_{s}=6$ the ED results exhibit larger finite-size ef- fects, as shown in Fig.17b). The variation with temperature is in this case also larger than for $n_{s}=8$. Evidently, the subtle features of spectral functions in the intermediate phase are not so well represented by including only two bath levels. Nevertheless, the qualitative difference between Fermi-liquid behavior for $J=0$ and the clear deviation from this behavior for $J=U / 4$ are very well reproduced by these $n_{s}=6 \mathrm{ED} / \mathrm{DMFT}$ calculations.

For completeness we show in Fig. 17(c) the $n_{s}=8$ ED/DMFT results for isotropic Hund's coupling, i.e., $J^{\prime}=J=U / 4$. This case is not yet accessible within QMC/DMFT because of sign problems at low temperatures. The inclusion of spin-flip and pair-exchange terms is seen to restore the limiting behavior $\Sigma_{2}\left(i \omega_{n}\right) \rightarrow 0$ for $\omega_{n} \rightarrow 0$. Nevertheless, compared to the case $J^{\prime}=J=0$ shown in (a), $\Sigma_{2}$ now increases much more rapidly at small finite frequencies, similarly to the data shown in Figs. 5 and 7 (As argued in Section 5, the shoulder near $\omega_{n} \approx 0.06$ is caused by finite-size effects.) Thus, instead of quasiparticles with weight $Z_{2} \approx 0.8$ for $J=J^{\prime}=0$, finite Hund's exchange with $J^{\prime}=J=U / 4$ supports a state of infinite lifetime at $E_{F}$, but does not satisfy Fermiliquid criteria at $\omega>0$. This picture is consistent with the $T=0 \mathrm{ED} / \mathrm{DMFT}$ results by Biermann et al. [21]. The spectral function of the wide band therefore should exhibit a narrow peak at $E_{F}$, similar to the one shown in Fig. 8 for $U=2.4 \mathrm{eV}$.

\section{COMMENTS ON IPT/DMFT RESULTS}

In Ref. 13] we reported DMFT calculations for the two-band Hubbard model within QMC and iterated perturbation theory [38]. Both approaches were shown to yield a consistent picture of the Mott transition, in the sense that both exhibit a single first-order transition at which the narrow band becomes insulating and the wide band begins to show progressive bad-metallic behavior. This band becomes fully insulating at a higher Coulomb energy in a non-first-order manner. As shown via ED/DMFT at $T=0$ in Ref. 14 and at $T>0$ in Ref. [16], the nature of this upper transition depends in a critical way on the treatment of onsite exchange interactions. In the absence of spin-flip and pair-exchange terms it is continuous, consistent with the QMC results, while for full Hund's coupling it becomes first-order.

The question then arises whether the IPT approach also supports this picture. In fact, as pointed out in Ref. [16], it is surprising that the IPT gives only one common first-order transition even though spin-flip and pair-exchange terms are included. It can easily be shown, however, that to second-order in the Coulomb interaction the subband self-energies with and without spin-flip and pair-exchange are the same, except for a slightly different relation between $U$ and $J$. Thus, $J^{\prime}=J=0.25 U$ gives the same result as $J^{\prime}=0$ and $J \approx 0.22 U$. Corrections to 
the simple second-order diagram via renormalization of subband energies to yield the correct atomic limit 37, 39. are also insensitive to the choice of $J^{\prime}$ for the present twoband model.

To resolve this puzzle we have repeated the IPT calculations reported in Ref. 13] at even lower temperatures and found indeed a tiny hysteresis loop also at the upper Mott transition, with a critical temperature of approximately $T_{c 2} \approx 5 \mathrm{meV}$, i.e., significantly lower than the critical temperature of the lower Mott transition, $T_{c 1} \approx 50 \mathrm{meV}$. These findings suggest that present formulations of IPT are too simple to deal with the full complexity of Hund's exchange. Diagrams beyond secondorder are required to distinguish more clearly between $J^{\prime}=J$ and $J^{\prime}=0$ treatments.

\section{SUMMARY AND OUTLOOK}

Finite-temperature ED/DMFT studies are carried out in order to explore the usefulness of this approach for multi-band systems. The important feature here is that onsite Coulomb and exchange interactions are fully included. Since in the past ED has been used mainly for single-band systems, a particular aim of this work is to illustrate the dependence of self-energies on the cluster size and to test the range of applicability of this method. As an example, we focus on the Hubbard model for two bands of different widths and investigate the metal insulator transition as a function of Hund's exchange.

The surprising and potentially very useful result of this work is that even a cluster size of $n_{s}=6$, with only two bath levels per impurity orbital, provides a qualitatively correct picture in all of the important phases of the $T / U$ phase diagram. Moreover, finite-size effects are substantially reduced for $n_{s}=8$, i.e., using one extra bath level per impurity orbital. Thus, in the phase just below the main first-order Mott transition, both subbands exhibit clear metallic properties, albeit with strongly reduced quasi-particle weights. The intermediate region between the purely metallic and insulating phases depends in a subtle manner on the exchange interactions included in the ED calculation. For full Hund's coupling coexisting metallic and insulating subbands are found, where the wide band exhibits infinite lifetime at $E_{F}$, but nonFermi-liquid behavior at finite frequencies. For Ising-like exchange, this breakdown of Fermi-liquid behavior is enhanced, giving finite lifetime even at $E_{F}$.

To explore the influence of finite-size effects in the twoband ED/DMFT approach NRG calculations were performed for an effective one-band model suitable to describe the wide band in the intermediate phase. Both for full Hund's coupling and Ising-like exchange, very good agreement with the ED results is found for temperatures in the range $T=20 \ldots 30 \mathrm{meV}$. At lower $T$, larger differences appear on a quantitative level at low frequen- cies. Nevertheless, the important qualitative differences between various phases, especially the characteristic lowfrequency variation of the self-energy in the two types of non-Fermi-liquid regions for isotropic and anisotropic exchange coupling, are fully reproduced by the ED approach, both for $n_{s}=6$ and $n_{s}=8$.

To test the accuracy of the two-band ED approach we also have applied it to models studied earlier within QMC/DMFT, neglecting spin-flip and pair-exchange. Nearly quantitative agreement is obtained for a cluster size $n_{s}=8$, but even the ED results for $n_{s}=6$ are found to be qualitatively reliable.

The present ED approach utilizes full diagonalization of the impurity Hamiltonian. Since at low temperatures only a limited range of excited states is relevant for the local Green's functions and self-energies it should be very useful to generalize finite- $T$ Lanczos one-band methods 22, 23 in order to bridge the gap between the present work and the true $T=0$ limit, and to apply the finitetemperature ED/DMFT approach to realistic two-band and three-band materials.

Acknowledgements: One of us (A. L.) likes to thank A. I. Lichtenstein for parts of the multi-band ED code. Some of the ED and NRG DMFT calculations were carried out on the IBM supercomputer (JUMP) of the Forschungszentrum Jülich.

Email: a.liebsch@fz-juelich.de; t.costi@fz-juelich.de

[1] For a review of early papers, see: A. Georges, G. Kotliar, W. Krauth and M. J. Rozenberg, Rev. Mod. Phys. 68, 13 (1996).

[2] For recent reviews, see: K. Held, cond-mat/0511293 A. Georges et al., cond-mat/0403123 G. Kotliar and S. Savrasov, cond-mat/0208241

[3] J. E. Hirsch and R. M. Fye, Phys. Rev. Lett. 56, 2521 (1986).

[4] For a discussion of this problem, see: K. Held and D. Vollhardt, Eur. Phys. J. B 5, 473 (1998).

[5] R. Arita and K. Held, Phys. Rev. B 96, 201102 (2005); see also: S. Sakai, R. Arita and H. Aoki, Phys. Rev. B 70, 172504 (2004).

[6] A. Koga, N. Kawakami, T. M. Rice, and M. Sigrist, Phys. Rev. B 72, 045128 (2005).

[7] See also: A. N. Rubtsov, V. V. Savkin, and A. I. Lichtenstein, Phys. Rev. B 72, 035122 (2005).

[8] M. Caffarel and W. Krauth, Phys. Rev. Lett. 72, 1545 (1994)

[9] V. I. Anisimov, I. A. Nekrasov, D. E. Kondakov, T. M. Rice and M. Sigrist, Euro. Phys. J. B 25, 191 (2002).

[10] A. Liebsch, Europhys. Lett. 63, 97 (2003).

[11] A. Liebsch, Phys. Rev. Lett. 91, 226401 (2003).

[12] A. Koga, N. Kawakami, T. M. Rice, and M. Sigrist, Phys. Rev. Lett. 92, 216402 (2004).

[13] A. Liebsch, Phys. Rev. B 70, 165103 (2004). 
[14] A. Koga, N. Kawakami, T. M. Rice, and M. Sigrist, Physica B 359-361, 1366 (2005).

[15] Yun Song and L.-J. Zou, Phys. Rev. B 72, 085114 (2005).

[16] A. Liebsch, Phys. Rev. Lett. 95, 116402 (2005).

[17] K. Inaba, A. Koga, S. Suga and N. Kawakami, J. Phys. Soc. Jpn. 74, 2393 (2005).

[18] L. de' Medici, A. Georges and S. Biermann, Phys. Rev. B 72, 205124 (2005).

[19] M. Ferrero, F. Becca, M. Fabrizio, and M. Capone, Phys. Rev. B 72, 205126 (2006).

[20] C. Knecht, N. Blümer, and P.G.J. van Dongen, Phys. Rev. B 72, 081103(R) (2005).

[21] S. Biermann, L. de' Medici, and A. Georges, Phys. Rev. Lett. 95, 206401 (2005).

[22] J. Jaklič and P. Prelovšek, Adv. Phys. 49, 1 (2000).

[23] M. Capone, L. de' Medici, and A. Georges, cond-mat/ 0512484

[24] T. Maeno, T. M. Rice, and M. Sigrist, Phys. Today 54, (1) 42 (2001).

[25] K. Takada et al., Nature 422, 53 (2003); R. E. Schaak et al., Nature 424, 527 (2003); M. L. Foo et al., Phys. Rev. Lett. 92, 247001 (2004).

[26] Th. Pruschke and R. Bulla, Eur. Phys. J. B 44, 217
(2005).

[27] T. A. Costi and N. Manini, J. Low Temp. Phys. 126, 835 (2002)

[28] K. G. Wilson, Rev. Mod. Phys. 47, 773 (1975).

[29] T. A. Costi, A. C. Hewson and V. Zlatic, J. Phys.: Condens. Matter 6, 2519 (1994).

[30] W. Hofstetter, Phys. Rev. Lett. 85, 1508 (2000).

[31] D. M. Cragg and P. Lloyd, J. Phys. C: Solid State Phys. 12, L215 (1979).

[32] W. Koller, A. C. Hewson and D. Meyer, Phys. Rev. B72, 045117 (2005).

[33] R. Bulla, T. Pruschke and A. C. Hewson, J. Phys.: Condens. Matter 10 , 8365 (1998).

[34] R. Bulla, T. A. Costi and D. Vollhardt, Phys. Rev. B64, 045103 (2001).

[35] T. A. Costi, to be published.

[36] A. Liebsch, to be published.

[37] H. Kajueter and G. Kotliar, Phys. Rev. Lett. 77, 131 (1997).

[38] A. Georges and G. Kotliar, Phys. Rev. B 45, 6479 (1992).

[39] A. I. Lichtenstein and M. I. Katsnelson, Phys. Rev. B 57, 6884 (1998). 